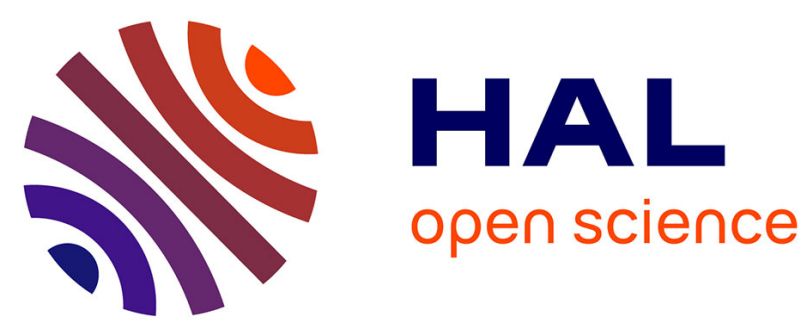

\title{
Listen to me! Public announcements to agents that pay attention - or not
}

Hans van Ditmarsch, Andreas Herzig, Emiliano Lorini, François

Schwarzentruber

\section{- To cite this version:}

Hans van Ditmarsch, Andreas Herzig, Emiliano Lorini, François Schwarzentruber. Listen to me! Public announcements to agents that pay attention - or not. 4th International Workshop on Logic, Rationality and Interaction (LORI IV), Oct 2013, Hangzhou, China. pp.96-109, 10.1007/978-3-64240948-6_8. hal-01187760

\section{HAL Id: hal-01187760 https://hal.science/hal-01187760}

Submitted on 27 Aug 2015

HAL is a multi-disciplinary open access archive for the deposit and dissemination of scientific research documents, whether they are published or not. The documents may come from teaching and research institutions in France or abroad, or from public or private research centers.
L'archive ouverte pluridisciplinaire HAL, est destinée au dépôt et à la diffusion de documents scientifiques de niveau recherche, publiés ou non, émanant des établissements d'enseignement et de recherche français ou étrangers, des laboratoires publics ou privés. 


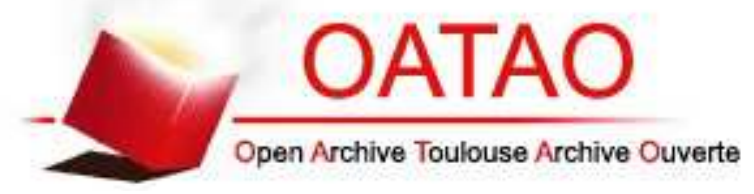

\section{Open Archive TOULOUSE Archive Ouverte (OATAO)}

OATAO is an open access repository that collects the work of Toulouse researchers and makes it freely available over the web where possible.

This is an author-deposited version published in : http://oatao.univ-toulouse.fr/ Eprints ID : 12651

To link to this article : doi: 10.1007/978-3-642-40948-6_8 URL : http://dx.doi.org/10.1007/978-3-642-40948-6_8

To cite this version : van Ditmarsch, Hans and Herzig, Andreas and Lorini, Emiliano and Schwarzentruber, François Listen to me! Public announcements to agents that pay attention - or not. (2013) In: 4th International Workshop on Logic, Rationality and Interaction (LORI IV), 9 October 2013 - 12 October 2013.

Any correspondance concerning this service should be sent to the repository administrator: staff-oatao@ listes-diff.inp-toulouse.fr 


\title{
Listen to Me! Public Announcements to Agents That Pay Attention — or Not
}

\author{
Hans van Ditmarsch ${ }^{1}$, Andreas Herzig ${ }^{2}$, \\ Emiliano Lorini ${ }^{2,3}$, and François Schwarzentruber ${ }^{4}$ \\ 1 LORIA, CNRS - Université de Lorraine, France \\ 2 University of Toulouse, CNRS, IRIT, France \\ 3 IAST, Toulouse, France \\ ${ }^{4}$ ENS Cachan (Brittany Extension), IRISA, France
}

\begin{abstract}
In public announcement logic it is assumed that all agents pay attention (listen to/observe) to the announcement. Weaker observational conditions can be modelled in event (action) model logic. In this work, we propose a version of public announcement logic wherein it is encoded in the states of the epistemic model which agents pay attention to the announcement. This logic is called attention-based announcement logic, abbreviated ABAL. We give an axiomatization and prove that complexity of satisfiability is the same as that of public announcement logic, and therefore lower than that of action model logic [2]. We exploit our logic to formalize the concept of joint attention that has been widely discussed in the philosophical and cognitive science literature. Finally, we extend our logic by integrating attention change.
\end{abstract}

\section{Introduction}

In public announcement logic it is assumed that announcements are perceived by all agents: it models the consequences of each of the agents incorporating a new formula into the set of beliefs. The argument of the dynamic modal operator in public announcement logic is therefore called an announcement. Once the government has announced a new election, they cannot be held liable when you forget to vote on election day. You were supposed to know.

In this work we take one step back from that point of view. When an announcement is made, it may well be that some agents were not paying attention and therefore did not hear it. Also, there may be uncertainty among the agents about who is paying attention and who not, and therefore, who heard the message and who not. Contrarily to action model logic, in our modelling it is not an aspect of the description of the action to which subset of all agents the announcement is made, but this is now an aspect of the state in which the announcement is executed.

Additional to the usual set of propositional variables we add designated variables for each agent, that express that the agent is paying attention. A given 
state of a Kripke model therefore contains information about which agents are paying attention and which agents are not paying attention. This determines the meaning of what we call attention-based announcements. A special case is that of introspective agents that know whether they are paying attention. We axiomatize our attention-based announcement logic $A B A L$, including a version with introspection for beliefs and attention.

An announcement by an outside observer that is public for a subset of all agents is modelled in $[9,6]$ as a private announcement to that subset of agents. The agents' attention configuration behind such announcements can be modelled in our logic by a particular formula built from our attention variables. Our logic generalizes Gerbrandy's because the 'attention level' of a given agent can vary between the states. Our logic can in turn be mapped to action model logic: each configuration of attention corresponds to a particular class of action models.

We show that the complexity of satisfiability in our logic remains in the same range as that of public announcement logic, viz. PSPACE. This contrasts with the higher complexity of action model logic. As the action models corresponding to attention-based announcements can be quite large, we consider that this is indeed a valuable result.

In the ABAL we can formalize a concept that has been widely discussed in the philosophical and in the cognitive science literature, namely joint attention $[15,13,7]$. This concept has been shown to be crucial for explaining the genesis of common belief in a group of agents.

Finally, we add other dynamics to our logic, namely change of attention. This is an elementary further addition to the logical framework and this logic also has a complete axiomatization.

\section{Attention-Based Announcement Logic ABAL}

Let $A G T$ be a finite set of agents, let $A T M$ be a (disjoint) countable set of propositional variables, and let $H=\left\{h_{a} \mid a \in A G T\right\}$ be a disjoint set of propositional variables. A proposition $h_{a}$ (for ' $a$ is hearing what is being said' or more simply ' $a$ is listening' ) expresses that agent $a$ is paying attention and so will hear public announcements.

Definition 1 (Language). The language $\mathcal{L}$ of attention-based announcement logic ABAL is defined as follows, where $p \in A T M$ and $a \in A G T$.

$$
\mathcal{L} \ni \quad \varphi::=p\left|h_{a}\right| \neg \varphi|(\varphi \wedge \varphi)| \mathbf{B}_{a} \varphi \mid[\varphi] \varphi
$$

We abbreviate $\bigwedge_{a \in A} h_{a}$ by $h_{A}$.

We write $q$ to denote a variable that is either $p \in A T M$ or $h_{a} \in H$. Other propositional connectives, and the dual modalities, are defined as usual. Formula $\mathbf{B}_{a} \varphi$ is read as 'agent $a$ believes that $\varphi$ is true', and formula $[\varphi] \psi$ as 'after the public announcement of $\varphi, \psi$ holds'. 
Definition 2 (Epistemic attention model). An epistemic attention model is a triple $M=(S, R, V)$ with $S$ a non-empty set, $R$ a function assigning to each agent an accessibility relation $R_{a}$ and $V$ a function assigning to each propositional variable $q \in A T M \cup H$ the subset $V(q) \subseteq S$ where the variable is true.

Definition 3 (Attention introspection). Given an epistemic attention model $M=(S, R, V)$, the model satisfies the property of attention introspection if for all $s, t \in S$, if $(s, t) \in R_{a}$, then $s \in V\left(h_{a}\right)$ iff $t \in V\left(h_{a}\right)$.

When attention introspection holds, an agent knows whether she is paying attention.

Boolean constructions as well as operators of belief $\mathbf{B}_{a}$ are interpreted in the standard way. The truth condition for attention-based announcements $[\varphi]$ is different from that of (world eliminating) truthful public announcement [14] and also different from that of (arrow eliminating) public announcement [9], although it comes closer to the latter in spirit: it is also arrow eliminating.

\section{Definition 4 (Semantics of attention-based announcements)}

$$
M, s \models[\varphi] \psi \text { iff } M^{\varphi},(s, 0) \models \psi
$$

where $M^{\varphi}=\left(S^{\prime}, R^{\prime}, V^{\prime}\right)$ is defined as follows.

$-S^{\prime}=S \times\{0,1\}$

- for each agent $a,((s, i),(t, j)) \in R_{a}^{\prime}$ if and only if $(s, t) \in R_{a}$ and:

1. $i=0, j=0,(M, s) \models h_{a}$ and $(M, t) \models \varphi$, or

2. $i=0, j=1$, and $(M, s) \not \models h_{a}$, or

3. $i=1, j=1$.

- for each $p \in A T M,(s, 0) \in V^{\prime}(p)$ iff $s \in V(p)$ and $(s, 1) \in V^{\prime}(p)$ iff $s \in V(p)$.

The model $M^{\varphi}$ is the extended disjoint union of the (arrows to) $\varphi$ restriction of $M$, called $M \mid \varphi$, and $M$ itself, plus - that is the extension - a number of additional accessibility pairs between states for those agents that are not attentive. Roughly speaking, $M^{\varphi}=M \mid \varphi \oplus M$ plus some edges. After the announcement of $\varphi$, the agents that are attentive only consider possible the 0 -copies of the states of the original model $M$ in which $\varphi$ is true. In contrast, the agents that are not attentive only consider possible 1-copies of the states of the original model $M$. This construction of the updated model $M^{\varphi}$ ensures that attentive agents learn $\varphi$ while inattentive agents don't learn anything.

Example 1. Ann $(a)$ and Bill (b) have lunch in the cafeteria and each consider the possibility of snowfall this afternoon $(p)$ - a regular occurrence in Nancy, many times of the year. In fact Bill has seen the weather report and knows whether it will snow, while Ann does not. However, Bill never knows whether Ann is paying attention. Ann knows that Bill is attentive. Both agents know whether they are attentive. This situation is depicted as model $M$ in Fig. 1 (there is no particular actual state: any of the four may do.) Now Cath comes along and says she just read the weather report: it will snow. This results in the 
model transition depicted in Figure 1, where Cath's announcement is modelled as an announcement by an outsider. Any of the four points in $M \mid p$ can be the actual state of the resulting model, but not any of the $M$ copy on the right hand side. If Ann and Bill pay attention and $p$ is true, as on the bottom-left side of $M \mid p$, Bill remains uncertain if Ann now knows that $p$, as he considers it possible that she was not paying attention (top-left of $M \mid p$ ), in which case she would have remained uncertain about $p$.

Proposition 1 (Preservation of attention introspection). If $M$ satisfies attention introspection then $M^{\varphi}$ satisfies attention introspection.

Although we consider Prop. 1 a valuable result, it takes somewhat away from the glamour when we realize that models with empty accessibility relations also satisfy attention introspection. For example, suppose that agent $a$ is paying attention in the actual state $s$ ( $h_{a}$ is true) and also in the (uniquely) accessible state $t$, and where $s$ is also considered possible. Attention introspection is satisfied. After the announcement with attentive agents $\neg h_{a}$, the agent $a$ no longer considers state $t$ possible but also no longer considers the actual state possible. Because the agent was paying attention, she has come to believe that she is not paying attention; but at the price of still also believing that she is paying attention - where the latter remains in fact the truth.

In this paper we focus on two classes of models: $\mathrm{K}_{n}$, where $n=|A G T|$, that is, multiple agents and no special properties of the accessibility relations, and $\mathrm{K}_{4} 5_{n}^{h}$, that is, multiple agents with transitive and Euclidean accessibility relations, and with attention introspection as well. (The classes $\mathrm{S} 5{ }_{n}^{h}$ and $\mathrm{KD} 45_{n}^{h}$ are unsuitable: they are not closed under announcements because the property of seriality $(D)$ may be lost after an announcement.) The set of valid $\mathcal{L}$-formulas on the class of models $\mathrm{K}_{n}$ is called ABAL, and the set of valid $\mathcal{L}$ formulas on the class of models $\mathrm{K} 45_{n}^{h}$ is called $\mathrm{ABAL}{ }^{\text {intro }}$.

\section{Relation with Action Models}

Every attention-based announcement is definable as an action model. Whether an announcement $\varphi$ is heard in a given state depends on the value of $h_{a}$ for every agent $a$ in that state. The agents who hear the announcement retain all arrows pointing to states where $\varphi$ holds and delete all arrows pointing to states where $\varphi$ does not hold, and that is independent of the truth of $\varphi$ in the actual state; whereas the agents who do not hear the announcement think that nothing has happened, i.e., also independent of the truth of $\varphi$ they think that the trivial action with precondition $T$ happened. There is an economic way to define such an action model (in the sense of producing a resulting model with a minimal duplication of states into bisimilar states). Definition 5 spells out the inductive clause for attention-based announcement of an obviously inductively defined translation. 

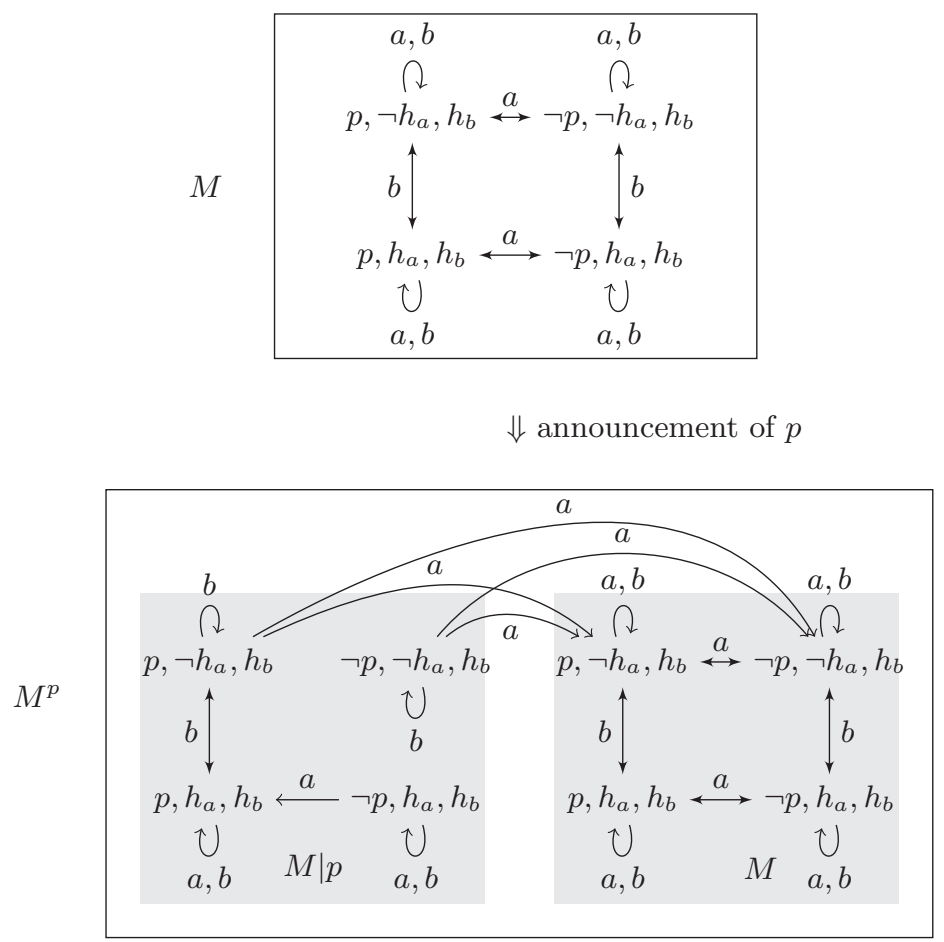

Fig. 1. Example of an attention-based announcement

Definition 5 (Action model for attention-based announcements). Given a formula $\varphi$, the action model for the attention-based announcement of $\varphi$ is the multi-pointed action model $\mathcal{A}_{\varphi}=(\mathbb{A}, R$, Pre,$P)$ where:

- $\mathbb{A}=\{(i, J) \mid i \in\{0,1\}$ and $J \subseteq A G T\} \cup\left\{w_{\top}\right\} ;$

$-R$ maps each agent $a \in A G T$ to

$$
\begin{aligned}
R_{a}= & \{((i, J),(1, K)) \mid i \in\{0,1\} \text { and } a \in J\} \cup \\
& \left\{\left((i, J), w_{\top}\right) \mid a \notin J\right\} \cup\left\{\left(w_{\top}, w_{\top}\right)\right\} ;
\end{aligned}
$$

- Pre $: \mathbb{A} \rightarrow \mathcal{L}$ is defined as follows:

- $\operatorname{Pre}((i, J))=\bar{\varphi} \wedge \bigwedge_{a \in A G T} \overline{h_{a}}$ where $\bar{\varphi}$ is either $\varphi$ if $i=1$ or $\neg \varphi$ if $i=0$ and $\overline{h_{a}}$ is either $h_{a}$ if $a \in J$ or $\neg h_{a}$ if $a \notin J$ for all $a \in A G T$;

- $\operatorname{Pre}\left(w_{\top}\right)=\top$;

- $P=\{(i, J) \mid i \in\{0,1\}$ and $J \subseteq A G T\}$ is the set of points.

Informally, the action model for the attention-based announcement of $\varphi$ consists of $2^{n+1}+1$ actions and has $2^{n+1}$ initial points (alias actual actions). Each of these points is identified by the complete and disjoint set of preconditions $\bar{\varphi} \wedge$ $\bigwedge_{a \in A G T} \overline{h_{a}}$, where $\bar{\varphi}$ is either $\varphi$ or $\neg \varphi$ and where $\overline{h_{a}}$ is either $h_{a}$ or $\neg h_{a}$. Moreover, there is a 'nothing happens' alternative with precondition $T$, that is not an 
initial point. An attentive agent believes that any action point with precondition entailing $\varphi$ may be the actual action. An inattentive agent believes that the action with precondition $T$ is the actual action.

The action model for attention-based announcements is depicted in Figure 2 for the example of two agents $a$ and $b$ and the announcement $\varphi$. For example, if $\varphi$ is false, $h_{a}$ is true, and $h_{b}$ is false, agent $a$ hears the announcement $\varphi$ and believes it to be true, therefore she believes the real action to be the one where $\varphi$ is true - regardless of the values of $h_{a}$ and $h_{b}$ in states wherein it can be executed (we take the case of general accessibility, i.e., logic $\mathrm{K}_{n}$ ). So that makes for four arrows. On the other hand, agent $b$ does not hear the announcement and believes that nothing at all happens: a single arrow to the alternative with precondition $T$ (and with reflexive arrows for $a$ and $b: b$ believes, incorrectly, that all agents believe that nothing happened).

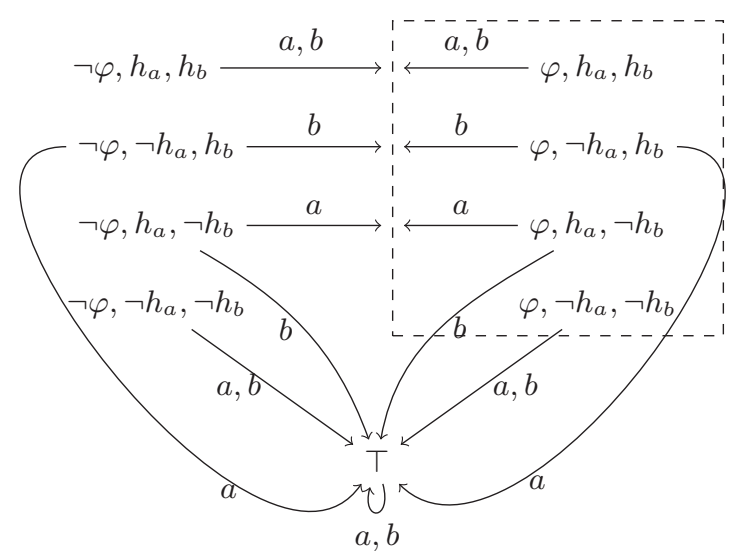

Fig. 2. The action model $\mathcal{A}$ corresponding to an attention-based announcements $\varphi$ to two agents. An arrow pointing to a box points to all actions in the box.

This action model construction maps nicely with the semantics of $[\varphi] \psi$ that given a model $M$ produces a model $M^{\varphi}$ twice that size, consisting of a 'trivial' copy $M$ plus a 'heard' copy $M \mid \varphi$. As all the $2^{n+1}$ different preconditions in the action model are exclusive, the product of that entire part of the action model produces a model of the same size as $M$, but with merely some removed arrows. A recursive translation defines an embedding from ABAL into action model logic.

Proposition 2. Let $M$ be an epistemic attention model. Let $\mathcal{A}_{\varphi}$ be the action

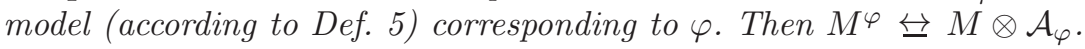

\section{Axiomatization and Complexity}

Table 1 shows the axiomatization. It follows the pattern of believed announcements (arrow eliminating), not that of truthful announcements (state eliminating). The crucial axiom is 


$$
[\varphi] \mathbf{B}_{a} \psi \leftrightarrow\left(\left(h_{a} \rightarrow \mathbf{B}_{a}(\varphi \rightarrow[\varphi] \psi)\right) \wedge\left(\neg h_{a} \rightarrow \mathbf{B}_{a} \psi\right)\right)
$$

It says that the belief consequences of an attention-based announcement are either, if the agent pays attention, what the agent believes to be the consequences of the announcement in case it was true, or else, if the agent does not pay attention, what the belief consequences were before the announcement (i.e., an agent not hearing the announcement does not change her beliefs). Note that our axiom resembles Gerbrandy's axiom for private announcements. The axioms * formalize that agents have introspective beliefs and are not uncertain about what they hear. (Attention introspection is therefore just like awareness introspection in a the logic of awareness [8].) The axiomatization ABAL consists of all the derivation rules and axioms of Table 1 . The axiomatization ABAL intro consists of ABAL plus the *-ed axioms and rules. Soundness follows straightforwardly from the action model modelling of attention-based announcements that we will give in Section 3.

Table 1. The axiomatizations for $A B A L$ and $A B A L^{\text {intro }}$

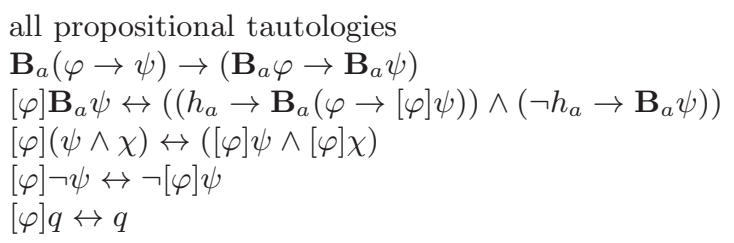

$$
\begin{aligned}
& \mathbf{B}_{a} \varphi \rightarrow \mathbf{B}_{a} \mathbf{B}_{a} \varphi \\
& \neg \mathbf{B}_{a} \varphi \rightarrow \mathbf{B}_{a} \neg \mathbf{B}_{a} \varphi \\
& h_{a} \rightarrow \mathbf{B}_{a} h_{a} \\
& \neg h_{a} \rightarrow \mathbf{B}_{a} \neg h_{a} \\
& \text { From } \varphi \text { infer } \mathbf{B}_{a} \varphi \\
& \text { From } \varphi \text { infer }[\psi] \varphi
\end{aligned}
$$

Proposition 3. The axiomatization of $A B A L$ is sound and complete for the class of $\mathrm{K}_{n}$ models. The axiomatization of $\mathrm{ABAL}{ }^{\text {intro }}$ is sound and complete for the class of $\mathrm{K}_{4} 5_{n}^{h}$ models.

Proof. The standard reduction argument applies: all axioms for the consequences of announcements push the announcement operator deeper into the formula on the right hand side, until one finally arrives at an announcement before a propositional variable, $[\varphi] p$, which is equivalent to $p$. Therefore the logic is equally expressive to the base modal logic — which is complete.

We note that by Proposition 1 the belief modality of $A B A L^{\text {intro }}$ does not collapse into a knowledge modality; see [5] for an investigation of this issue.

In the remainder of the section we focus on the ABAL satisfiability problem on the class of all $\mathrm{K}_{n}$ frames. The satisfiability problem of a formula in the language of action model logic [6] plus the union operator over actions is NEXPTIMEcomplete [2]. ABAL is the fragment of action model logic for an action model of size exponential in the number of agents (see Section 3). So the satisfiability problem of ABAL is decidable.

It is difficult to turn the tableau method for action model logic into a PSPACE procedure because each node may contain an exponential amount of information in the length of the input formula. Surprisingly, we can adapt the tableau method 
of action model logic so that the amount of information in a node is polynomial in the size of the input.

Proposition 4. Satisfiability of $\mathcal{L}$ formulas in the class of $\mathrm{K}_{n}$ models is PSPACE-complete.

We leave open the complexity of satisfiability of formulas of the ABAL on the class of $\mathrm{K}_{4}{ }_{n}^{h}$ models. We conjecture that it equals that of the underlying epistemic logic, viz. PSPACE complete.

\section{Joint Attention}

The attention introspection axiom $h_{a} \rightarrow \mathbf{B}_{a} h_{a}$ of $\mathrm{ABAL}^{\text {intro }}$ only guarantees attention introspection for individuals, not for groups: it may happen that $h_{A}$ is true while some $a \in A$ does not believe that $h_{A}$. We now investigate a condition under which attention introspection obtains in terms of common belief: joint attention or joint attentional state. That concept was widely discussed in the philosophical and in the cognitive science literature $[15,13,7]$, and we show that it can be captured in our logic $A B A L^{\text {intro }}$.

We assume a common belief operator $\mathbf{C}_{A}$ for a subgroup $A$ of the set of all agents, so that $\mathbf{C}_{A} \varphi$ stands for 'the agents in group $A$ commonly believe $\varphi$ ', and which is interpreted in the usual way by the transitive closure of the union of all accessibility relations $R_{a}$ for the agents in $A$.

Let $A \subseteq A G T$. The idea is that the agents in $A$ have a joint attention (or are in a joint attentional state) if and only if they are looking at the source of information together, that is to say, every agent in $A$ is looking at the source of information, every agent in $A$ believes that every agent in $A$ is looking at the source of information, and so on. More concisely, the agents in $A$ are in a joint attentional state if and only if each of them is looking at the source of information and focusing his attention on it and they have common belief that each of them is looking at the source of information and focusing his attention on it. Formally:

$$
\text { JointAtt } \equiv_{\text {def }} h_{A} \wedge \mathbf{C}_{A} h_{A} .
$$

Note that joint attention is closed under attention-based announcements: if $M, s \models \operatorname{JointAtt}_{A}$ then $M^{\varphi}, s \models \operatorname{Joint} \mathrm{Att}_{A}$ for every $\varphi$ such that $M, s \models \varphi$. Note moreover that when joint attention of all agents is satisfied then attention-based announcements are the same as public announcements.

As pointed out by $[13,7]$, joint attention explains the genesis of common beliefs in the context of social interaction. Such genesis is often considered as related to public events in the sense that a common belief is either a consequence of an event whose occurrence is so evident (viz. public) that agents cannot but recognize it as when, during a soccer match, players mutually believe that they are playing soccer, or the product of a communication process as when the referee publicly announces that one player is expelled. From there on each player believes that each other player believes and so on that one of them has been 
expelled. Intuitively, an event is considered public as long as its occurrence is epistemically accessible by everybody such that it becomes common belief between them. But what are the intuitive conditions that make an event public? What are the reasons to believe that an occurring event is commonly believed? In a normal situation (what is announced is true, there is no noise in the communication channel, etc.) looking at the source of information and having a common belief that everyone is looking at the source of information (i.e., being in a joint attentional state) provide a sufficient condition for the formation of a common belief. This is captured by a validity of ABAL (to avoid Moorean phenomena we restrict ourselves to learning propositional variables):

$$
\models \operatorname{Joint} \mathrm{Att}_{A} \rightarrow[p] \mathbf{C}_{A} p
$$

We can actually characterize the formation of common belief of an atomic fact $p$ as follows:

$$
\models[p] \mathbf{C}_{A} p \leftrightarrow \mathbf{C}_{A} \bigwedge_{a \in A}\left(h_{a} \vee \mathbf{B}_{a} p\right)
$$

Note that the equivalence is not valid if we replace $p$ by $h_{a}$.

\section{Attention Change}

A good way to have your addressees pay attention is to clap your hands before making an announcement. Even if they were not paying attention, they now do. In other words, if $\neg h_{a}$ was true before, $h_{a}$ is true now. And this is the case for all agents. This is a public way to make everybody pay attention to you. Even more, you have achieved their joint attention.

A less public way to make someone listen to you is to tap on her shoulder before you speak. This only makes that person attentive and not the other agents. Suppose that agents $a$ and $b$ are both not paying attention. If I tap on $a$ 's shoulder and then say something, only $a$ and not $b$ will hear it. If, on the other hand, I first tap $a$ 's shoulder and then $b$ 's then both $a$ and $b$ will hear the announcement, but they consider it possible that the other was not paying attention and does not hear it. The order does not make a difference. In contrast, clapping your hands is a way to ensure joint attention.

Drawing inspiration from $[17,16]$, we model such fine-grained attention change by an assignment. Given a set of agents $A \subseteq A G T$, we distinguish the assignment $+A$ (merely shorthand for a simultaneous assignment $a_{1}:=\top, \ldots, a_{n}:=\top$ ) that makes all agents $a \in A$ pay attention and hear subsequent announcements, from an assignment $-A$ that makes all $h_{a}$ false. To the inductive definition of the language $\mathcal{L}$ (Def. 1 ) we add clauses for the modal operators $[+A]$ and $[-A]$, for $A \subseteq A G T$. We write $\left[+a_{1}, \ldots, a_{n}\right]$ instead of $\left[+\left\{a_{1}, \ldots, a_{n}\right\}\right]$. The semantics of attention-based assignment is then:

$$
\begin{aligned}
& M, s \models[+A] \psi \text { iff } M^{+A},(s, 0) \models \psi \\
& M, s \models[-A] \psi \text { iff } M^{-A},(s, 0) \models \psi
\end{aligned}
$$

where $M^{+A}=\left(S^{\prime}, R^{\prime}, V^{\prime}\right)$ is defined as follows (the definition of $M^{-A}$ is similar). 
$-S^{\prime}=S \times\{0,1\}$

- if $a \in A$ and $s, t \in S$ then $((s, i),(t, j)) \in R_{a}^{\prime}$ iff $(s, t) \in R_{a}$ and

1. (1) $i=0$ and $j=0$; or

2. (2) $i=1$ and $j=1$;

- if $a \notin A$ and $s, t \in S$ then $((s, i),(t, j)) \in R_{a}^{\prime}$ iff $(s, t) \in R_{a}$ and

1. (1) $i=0, j=0$, and $(M, s) \models h_{a}$; or

2. (2) $i=0, j=1$, and $(M, s) \not \forall h_{a}$; or

3. (3) $i=1$ and $j=1$;

- $(s, 0) \in V^{\prime}(p)$ iff $s \in V(p)$, and $(s, 1) \in V^{\prime}(p)$ iff $s \in V(p)$;

- if $a \in A$ then

1. $(s, 0) \in V^{\prime}\left(h_{a}\right)$ iff $s \in V\left(h_{a}\right)$, and

2. $(s, 1) \in V^{\prime}\left(h_{a}\right)$ iff $s \in V\left(h_{a}\right)$;

- if $a \notin A$ then

1. $(s, 0) \in V^{\prime}\left(h_{a}\right)$, and

2. $(s, 1) \in V^{\prime}\left(h_{a}\right)$ iff $s \in V\left(h_{a}\right)$.

In the case of the singleton attention assignment $+a$, agent $a$ will now pay attention in the 0-copy of the initial model and may or may not be paying attention in the 1-copy (that copies the prior information state). If another agent $b$ was already paying attention he will now know that $a$ is now paying attention (the first item of the clause for agents not paying attention, above, wherein arrows point to other 0-worlds); else his knowledge of $a$ 's attention span is as before (the second item of the clause for agents not paying attention). The only factual information change takes place in the 0-copy, and only for $h_{a}$ (this is the part $(s, 0) \in V^{\prime}\left(h_{a}\right)$ in the last item, i.e., for all states $s$ in the 0-copy $h_{a}$ is now true).

Attention assignment preserves attention introspection. The order in successive change of attention does not matter, but it does not achieve joint attention:

$-\models[+a][+b] \varphi \leftrightarrow[+b][+a] \varphi$

$-\not \forall[+a, b] \varphi \leftrightarrow[+b][+a] \varphi$.

Just as attention-based announcements, an attention assignment correspond to an action model that is a function of (1) who is paying attention and who not before the assignment, and (2) the assignment. The logic to which attention assignment has been added can therefore also easily be axiomatized. (Details of this action model are omitted.)

The validities of the language extended by assignments can be axiomatized by means of reduction axioms for $[+A]$ and $[-A]$. Separate axioms are needed for all inductive cases $[+A] \varphi$ (again, just as for attention-based announcement, as a function of a rather complex action model). For the case of epistemic operators these are:

$$
\begin{aligned}
& {[+A] \mathbf{B}_{a} \varphi \leftrightarrow \begin{cases}\mathbf{B}_{a}[+A] \varphi & \text { if } a \in A \\
\left(h_{a} \rightarrow \mathbf{B}_{a}[+A] \varphi\right) \wedge\left(\neg h_{a} \rightarrow \mathbf{B}_{a} \varphi\right) & \text { if } a \notin A\end{cases} } \\
& {[-A] \mathbf{B}_{a} \varphi} \\
& {\left[\begin{array}{ll}
\mathbf{B}_{a}[-A] \varphi & \text { if } a \in A \\
\left(h_{a} \rightarrow \mathbf{B}_{a}[-A] \varphi\right) \wedge\left(\neg h_{a} \rightarrow \mathbf{B}_{a} \varphi\right) & \text { if } a \notin A
\end{array}\right.}
\end{aligned}
$$


Finally, let $[[\varphi]]_{A}$ be the modal operator of private announcement to group $A$ according to [9]. Then $[[\varphi]]_{A} \psi$ is equivalent to $[+A][-\bar{A}][\varphi] \psi$, where $\bar{A}$ is $A G T \backslash A$. It follows that the public announcement of $\varphi$ can be captured in our logic by $[+A G T][\varphi]$.

\section{Comparison and Further Research}

Our proposal is related to several other logics in the DEL literature: arrow update logic [12], wherein a simple dynamic operator can have a large action model equivalent; reasoning about perception [18]; reasoning about perceptual beliefs [11]; reasoning about visually oriented agents [3]; action languages [1].

We plan to study the extension of attention-based announcement logic with common belief that we have sketched in Section 5. On a similar setting we intend to model trust-based announcement logic.

Acknowledgements. We thank the LORI reviewers for their comments and high praise. Hans van Ditmarsch is also affiliated to IMSc, Chennai, as research associate. We acknowledge support from European Research Council grant EPS 313360. Andreas Herzig and Emiliano Lorini acknowledge support of the the ANR project EmoTES.

\section{References}

1. Aucher, G.: BMS revisited. In: Proc. of 12th TARK, pp. 24-33 (2009)

2. Aucher, G., Schwarzentruber, F.: On the complexity of dynamic epistemic logic. In: Proc. of 13th TARK (2013)

3. Balbiani, P., Gasquet, O., Schwarzentruber, F.: Agents that look at one another. Logic Journal of IGPL (2012)

4. Balbiani, P., van Ditmarsch, H., Herzig, A., De Lima, T.: Tableaux for public announcement logic. Journal of Logic and Computation 20(1), 55-76 (2010)

5. Balbiani, P., van Ditmarsch, H., Herzig, A., de Lima, T.: Some truths are best left unsaid. In: Advances in Modal Logic (AiML). College Publications (2012)

6. Baltag, A., Moss, L.S., Solecki, S.: The logic of public announcements, common knowledge, and private suspicions. In: Proc. of 7th TARK, pp. 43-56 (1998)

7. Clark, H.H., Marshall, C.R.: Definite reference and mutual knowledge. In: Elements of Discourse Understanding. Cambridge University Press (1981)

8. Fagin, R., Halpern, J.Y.: Belief, awareness, and limited reasoning. Artificial Intelligence 34(1), 39-76 (1987)

9. Gerbrandy, J.D.: Bisimulations on Planet Kripke. PhD thesis, University of Amsterdam, ILLC Dissertation Series DS-1999-01 (1999)

10. Halpern, J.Y., Moses, Y.: A guide to completeness and complexity for modal logics of knowledge and belief. Artificial Intelligence 54, 319-379 (1992)

11. Herzig, A., Lorini, E.: A modal logic of perceptual belief. In: Lihoreau, F., Rebuschi, M. (eds.) Epistemology, Context and Formalism. Synthese Library. Springer (2013)

12. Kooi, B., Renne, B.: Arrow update logic. The Review of Symbolic Logic 4(04), 536-559 (2011) 
13. Lorini, E., Tummolini, L., Herzig, A.: Establishing mutual beliefs by joint attention: towards a formal model of public events. In: Proc. of CogSci, pp. 1325-1330. Lawrence Erlbaum Associates (2005)

14. Plaza, J.A.: Logics of public communications. In: Proc. of the 4th ISMIS, pp. 201-216. Oak Ridge National Laboratory (1989)

15. Tommasello, M.: Joint attention as social cognition. In: Moore, C., Dunham, P. (eds.) Joint Attention: its Origins and Role in Development, pp. 103-130. Lawrence Erlbaum Associates (1995)

16. van Benthem, J., van Eijck, J., Kooi, B.: Logics of communication and change. Information and Computation 204(11), 1620-1662 (2006)

17. van Ditmarsch, H., van der Hoek, W., Kooi, B.: Dynamic epistemic logic with assignment. In: Proc. of 4th AAMAS, pp. 141-148. ACM (2005)

18. van Eijck, J.: Perception and change in update logic. In: van Eijck, J., Verbrugge, R. (eds.) Games, Actions and Social Software 2010. LNCS, vol. 7010, pp. 119-140. Springer, Heidelberg (2012)

\section{Annex: Tableaux and Complexity}

Let $\mathfrak{L} \mathfrak{a} \mathfrak{b}$ be a countable set of labels designed to represent worlds of the epistemic model $(M, w)$. Our tableau method manipulates terms that we call tableau terms. They are of the following kind:

- $(\sigma \Sigma \varphi)$ where $\sigma \in \mathfrak{L} \mathfrak{a b}$ is a symbol (that represents a world in the initial model) and $\Sigma$ is a sequence of formulas (where [] denotes the empty list). This term means that $\varphi$ is true after the announcements of the formulas of the sequence $\Sigma$ (in that order) in the world denoted by $\sigma$;

- $(\sigma \Sigma \checkmark)$ means that the sequence $\Sigma$ is executable in $\sigma$;

- $(\sigma \Sigma \otimes)$ means that the sequence $\Sigma$ is not executable in the world denoted by $\sigma$;

- $\left(\sigma R_{a} \sigma_{1}\right)$ means that the world denoted by $\sigma$ is linked by $R_{a}$ to the world denoted by $\sigma_{1}$;

$-\perp$ denotes an inconsistency.

A tableau rule is represented by a numerator $\mathcal{N}$ above a line and a finite list of denominators $\mathcal{D}_{1}, \ldots, \mathcal{D}_{k}$ below this line, separated by vertical bars:

$$
\frac{\mathcal{N}}{\mathcal{D}_{1}|\ldots| \mathcal{D}_{k}}
$$

The numerator and the denominators are finite sets of tableau terms.

A tableau tree is a finite tree with a set of tableau terms at each node. A rule with numerator $\mathcal{N}$ is applicable to a node carrying a set $\Gamma$, if $\Gamma$ contains an instance of $\mathcal{N}$. If no rule is applicable, $\Gamma$ is said to be saturated. We call a node $\sigma$ an end node, if the set of formulas $\Gamma$ it carries is saturated or if $\perp \in \Gamma$. The tableau tree is extended as follows:

1. Choose a leaf node $n$ carrying $\Gamma$ where $n$ is not an end node, and choose a rule $\rho$ applicable to $n$. 
2. (a) If $\rho$ has only one denominator, add the appropriate instantiation to $\Gamma$.

(b) If $\rho$ has $k$ denominators with $k>1$, create $k$ successor nodes for $n$, where each successor $i$ carries the union of $\Gamma$ with an appropriate instantiation of denominator $\mathcal{D}_{i}$.

A branch in a tableau tree is a path from the root to an end node. A branch is closed if its end node contains $\perp$, otherwise it is open. A tableau tree is closed if all its branches are closed, otherwise it is open. The tableau tree for a formula $\varphi \in \mathcal{L}$ is the tableau tree obtained from the root $\left\{\left(\sigma_{0}[] \varphi\right)\right\}$ when all leaves are end nodes.

The tableau rules are depicted in Figure 3. They contain the classical Boolean rules $(\wedge),(\neg \neg)$ and a non-deterministic rule $(\neg \wedge)$ handling disjunctions. The rule $(\perp)$ makes the current execution fail. The rules $\left(\leftarrow_{p}\right)$ and $\left(\leftarrow_{\neg p}\right)$ correspond to the fact that valuations are not changed by announcements. The rule (hear) decides non-deterministically for all atomic propositions $h_{a}$ whether they are true or false. Note that this is a non-analytic rule: the formulas in its denominator are not necessarily subformulas of the input formula. Depending on the value of $h_{a}$, there are two versions of the rule for $\mathbf{B}_{a}$ and for $\neg \mathbf{B}_{a}$. The rules $(\checkmark),(\otimes)$, $\left(\operatorname{clash}_{\checkmark, \otimes}\right)$ and $\left(\epsilon_{\otimes}\right)$ deal with executability of the sequence $\Sigma$.

Proposition 5 (Soundness and Completeness of the Tableau Method). $A \mathcal{L}$ formula $\varphi$ is satisfiable iff there exists an open branch for $\varphi$.

Proof. $\Leftarrow$ If the formula $\varphi$ is satisfiable, there exists a pointed model $(M, w)$ such that $M, w \models \varphi$. We use the model $M$ and the updated models from $M$ and the announcement in $\varphi$ as an oracle to guide the execution of the tableau method yielding to an open branch.

$\Rightarrow$ Given an open branch, we construct a model $M$ where worlds are the nodes $\sigma$, relations are inferred from terms of the form $\left(\sigma R_{a} \sigma_{1}\right)$ and valuations are inferred from terms of the form $(\sigma \epsilon p)$ and $(\sigma \epsilon \neg p)$. We prove by induction over $\Sigma, \psi$ that $(\sigma \Sigma \psi)$ is in the branch iff $M^{\Sigma}, \sigma \models \psi$, where $M^{\Sigma}$ is the model obtained by updating $M$ by the sequence $\Sigma$.

Proposition 6. Satisfiability of $\mathcal{L}$ formulas in the class of $\mathrm{K}_{n}$ models is PSPACE-complete.

Proof. As explained in [10], a tableau method leads to a PSPACE procedure if we can apply the rules by only keeping in memory the content of a branch. In our case the argument is essentially the same (see also [4]): we only keep in memory the information concerning the current node and its path to the root node, in order to be able to backtrack. We implicitly restrict the applicability of the (hear) rule to those $h_{a}$ such that $a$ occurs in the input formula. It is PSPACE-hard because we may reduce polynomially the satisfiability problem for $\mathrm{K}_{n}$ (the multi-agent version of the minimal modal logic $\mathrm{K}$ ). 


$$
\begin{aligned}
& \frac{(\sigma \Sigma \varphi \wedge \psi)}{(\sigma \Sigma \varphi)}(\wedge) \quad \frac{(\sigma \Sigma \neg \neg \varphi)}{(\sigma \Sigma \Sigma)}(\neg \neg) \\
& \frac{(\sigma \Sigma \neg(\varphi \wedge \psi))}{(\sigma \Sigma \neg \varphi) \mid(\sigma \Sigma \neg \psi)}(\neg \wedge) \quad \frac{(\sigma \Sigma p)(\sigma \Sigma \neg p)}{\perp}(\perp) \\
& \frac{(\sigma \Sigma[\varphi] \psi)}{(\sigma \Sigma:: \varphi \psi)}([\varphi]) \quad \frac{(\sigma \Sigma \neg[\varphi] \psi)}{(\sigma \Sigma: \varphi \neg \psi)}(\neg[\varphi]) \\
& \frac{(\sigma \Sigma p)}{(\sigma[] p)}\left(\leftarrow_{p}\right) \quad \frac{(\sigma \Sigma \neg p)}{(\sigma[] \neg p)}(\leftarrow \neg p)
\end{aligned}
$$

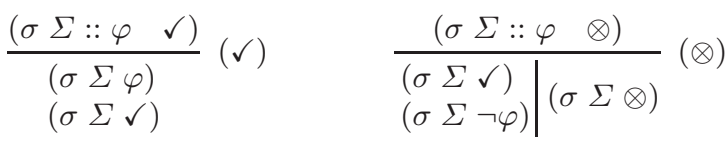

$$
\begin{aligned}
& \frac{(\sigma \Sigma \otimes)(\sigma \Sigma \checkmark)}{\perp}\left(\operatorname{clash}_{\checkmark, \otimes}\right) \quad \frac{(\sigma[] \otimes)}{\perp}\left([]_{\otimes}\right) \\
& \left(\sigma \Sigma \mathbf{B}_{a} \varphi\right)\left(\sigma[] h_{a}\right)
\end{aligned}
$$

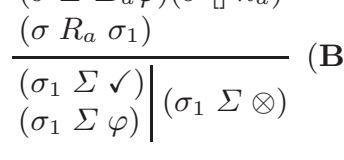

$$
\begin{aligned}
& \left(\sigma \Sigma \mathbf{B}_{a} \varphi\right)\left(\sigma[] \neg h_{a}\right) \\
& \frac{\left(\sigma R_{a} \sigma_{1}\right)}{\left(\sigma_{1}[] \varphi\right)}\left(\mathbf{B}_{a}\right) \\
& \begin{array}{cc}
\frac{\left(\sigma[] h_{a}\right)\left(\sigma \Sigma \neg \mathbf{B}_{a} \varphi\right)}{\left(\sigma R_{a} \sigma_{\text {new }}\right)}\left(\neg \mathbf{B}_{a}\right) & \frac{\left(\sigma[] \neg h_{a}\right)\left(\sigma \Sigma \neg \mathbf{B}_{a} \varphi\right)}{\left(\sigma R_{a} \sigma_{\text {new }}\right)} \\
\left(\sigma_{\text {new }} \Sigma \checkmark\right) & \left(\neg \mathbf{B}_{a}\right) \\
\left(\sigma_{\text {new }} \Sigma \neg \varphi\right) & \left(\sigma_{\text {new }}[] \neg \varphi\right)
\end{array} \\
& \overline{\left(\sigma[] h_{a}\right) \mid\left(\sigma[] \neg h_{a}\right)} \text { (hear) }
\end{aligned}
$$

Fig. 3. Tableau rules 